\title{
Measurement of synchrotron-radiation-excited Kossel patterns
}

\author{
G. Bortel, G. Faigel, M. Tegze and A. Chumakov
}

J. Synchrotron Rad. (2016). 23, 214-218

\section{IUCr Journals CRYSTALLOGRAPHY JOURNALS ONLINE}

Copyright (C) International Union of Crystallography

Author(s) of this paper may load this reprint on their own web site or institutional repository provided that this cover page is retained. Republication of this article or its storage in electronic databases other than as specified above is not permitted without prior permission in writing from the IUCr.

For further information see http://journals.iucr.org/services/authorrights.html 


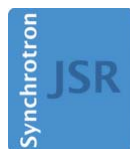

JOURNAL OF SYNCHROTRON RADIATION

ISSN $1600-5775$

Received 15 July 2015

Accepted 9 October 2015

Edited by A. Momose, Tohoku University, Japan

Keywords: Kossel pattern; fluorescence; diffraction.

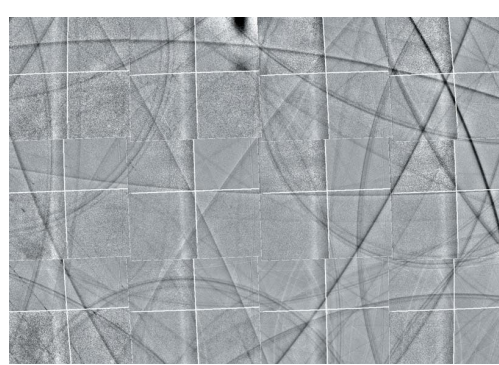

C 2016 International Union of Crystallography

\section{Measurement of synchrotron-radiation-excited Kossel patterns}

\author{
G. Bortel, ${ }^{a} *$ G. Faigel, ${ }^{a}$ M. Tegze ${ }^{a}$ and A. Chumakov ${ }^{b}$
}

aWigner Research Centre for Physics, Institute for Solid State Physics and Optics, POB 49, H-1525 Budapest,

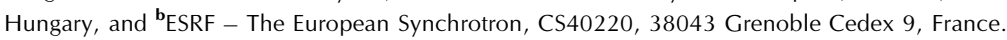

*Correspondence e-mail: bortel.gabor@wigner.mta.hu

Kossel line patterns contain information on the crystalline structure, such as the magnitude and the phase of Bragg reflections. For technical reasons, most of these patterns are obtained using electron beam excitation, which leads to surface sensitivity that limits the spatial extent of the structural information. To obtain the atomic structure in bulk volumes, X-rays should be used as the excitation radiation. However, there are technical problems, such as the need for high resolution, low noise, large dynamic range, photon counting, twodimensional pixel detectors and the small spot size of the exciting beam, which have prevented the widespread use of Kossel pattern analysis. Here, an experimental setup is described, which can be used for the measurement of Kossel patterns in a reasonable time and with high resolution to recover structural information.

\section{Introduction}

Traditionally, single-crystal X-ray diffraction is used to determine atomic order in solids. However, in the case of crystals which contain atoms emitting fluorescent radiation in the $\mathrm{X}$-ray regime, similar information can be obtained from Kossel line patterns (Kossel et al., 1935; Lider, 2011). For an easier understanding of the formation of Kossel lines, see the drawing given in Fig. 1. An excited atom emits a fluorescent photon in the de-excitation process, which travels as an outgoing spherical wave. Part of this wave is scattered by the

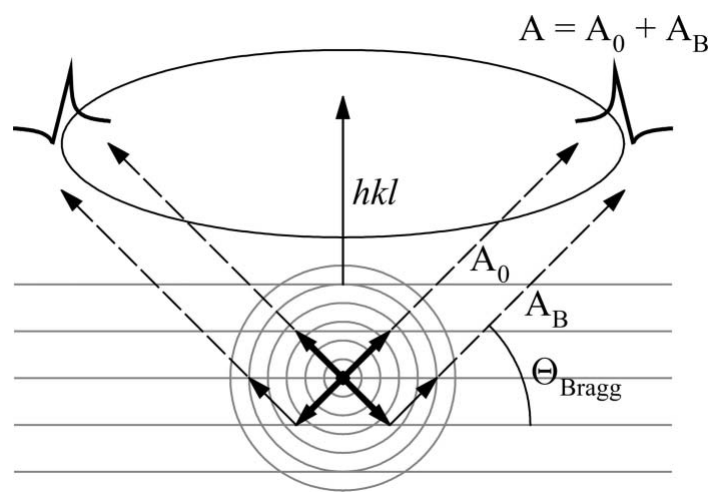

Figure 1

Formation of Kossel lines. Fluorescent radiation emitted by the atoms $\left(A_{0}\right)$ in the sample interferes with its Bragg reflection $\left(A_{\mathrm{B}}\right)$ resulting in strong intensity modulations $\left(A=A_{0}+A_{\mathrm{B}}\right)$ in the vicinity of cones defined by the normal of the diffracting plane $(h k l)$ and the Bragg angle $\left(\Theta_{\text {Bragg }}\right)$. The viewing direction is in the diffracting planes (grey lines) but the cone is illustrated with an ellipse. Arrows indicate relevant direct (thick arrows) and reflected (thin arrows) plane-wave components of the spherical-wave fluorescent radiation. 
crystal lattice, while the other part reaches the detector without scattering. These two waves interfere and this interference pattern is recorded by the detector. It is clear from the geometry that the Bragg condition is satisfied for cones having axes perpendicular to the various crystalline planes and opening angles $180^{\circ}-2 \Theta_{\text {Bragg. The strongest intensity varia- }}$ tion due to interference is observable in the vicinity of these cones. Different phase relations of the reflected and the direct waves result in different angular dependences of the intensity in this region; hence, the Kossel line profiles depend on the phase of the corresponding Bragg reflection (Cowley, 1964; Hutton et al., 1985).

Compared with single-crystal diffraction, Kossel patterns have at least two advantages: (i) they can directly give not only the magnitude of the reflections as traditional diffraction but also the phase (Cowley, 1964; Hutton et al., 1985); (ii) a pattern containing many Kossel lines can be taken at a single orientation of the sample, i.e. there is no need to rotate the sample as in traditional diffraction experiments. The possibility of phase determination is unique and would significantly facilitate structure determination. The second feature is useful in special sample environments, such as in studies at high temperature, in high-magnetic field or for light-excited processes, etc., where the stationary sample makes these measurements technically much easier. The stationary sample also makes it possible for short data collection times; a pattern containing complete structural information could be taken in seconds at a synchrotron source or in the extreme case in femtoseconds at a free-electron laser. These features would make this type of experiment ideal for many kind of studies.

In spite of the above-mentioned advantages, Kossel pattern measurements are not common. Mostly they are performed in special cameras or in electron microscopes (Lider, 2011) using an electron beam as the primary excitation source to generate X-ray fluorescence. (Here the Kossel pattern created by X-rays should not be confused with the Kikuchi pattern created by electrons.) Electron excitation leads to surface sensitivity and a continuum background caused by the Bremsstrahlung radiation beside the pattern forming the characteristic fluorescent lines. An advantage of electron excitation is good decoupling of the probe beam (electrons) from the pattern forming waves (X-rays). The surface sensitivity means that we need a clean surface, otherwise we cannot obtain bulk structural information. If we want to obtain bulk structural information on the scale of a few micrometres or larger, we have to use X-rays for the primary excitation of the fluorescent radiation. However, in this case the exciting radiation is not decoupled so well from the pattern itself, as both are X-rays in the same, typically $\sim 10 \mathrm{keV}$, energy range, leading to high background and strong Bragg reflections in certain orientations that may mask the Kossel pattern. On the other hand, a practical advantage of X-ray excitation is the absence of the continuous Bremsstrahlung background radiation.

In this paper, we describe an experimental setup that is capable of taking synchrotron-radiation-excited Kossel patterns in reasonable time and with high enough resolution to recover structural information. The experiment utilizes developments in synchrotron source optics (Kirkpatrick \& Baez, 1948), two-dimensional X-ray pixel detectors (Hatsui \& Graafsma, 2015) and elaborated evaluation procedures.

\section{Design considerations}

A sketch of our experiment is shown in Fig. 2. There are two critical components in the system, the focusing mirror and the two-dimensional pixel detector. In order to understand why these units are essential in these experiments, let us discuss the various requirements.

From the theory of dynamical diffraction, we know that the fine structure of Kossel lines containing the phase information is in the arcsecond range (Cowley, 1964; Hutton et al., 1985), requiring very high angular resolution. Assuming practical sample-to-detector distances, this translates to a spatial resolution in the $10 \mu \mathrm{m}$ range, limiting both the spot size of the probe beam and the pixel size of the detector.

Interference of the direct outgoing fluorescent radiation and its reflection modulates the intensity in the vicinity of the cones satisfying the Bragg condition. Theoretically for this modulation, the fine structure of the Kossel lines is on the scale 0-4 relative to the fluorescent radiation: for fully constructive interference it is 4, for completely destructive interference it is 0 (Cowley, 1964; Hutton et al., 1985). However, in practice this contrast is much lower. The reason is mostly the non-perfect nature of the crystals. This smears out the Kossel line profiles, increasing their characteristic width and decreasing the intensity modulation. On one hand this relaxes the pixel size requirement but it requires a large dynamic range for the detector.

Another factor affecting the quality of the obtainable Kossel patterns is the suppression of the primary exciting $\mathrm{X}$-ray radiation relative to the fluorescent X-ray radiation. This can be achieved by proper shielding, eliminating airscattering and by using detectors with good energy separation

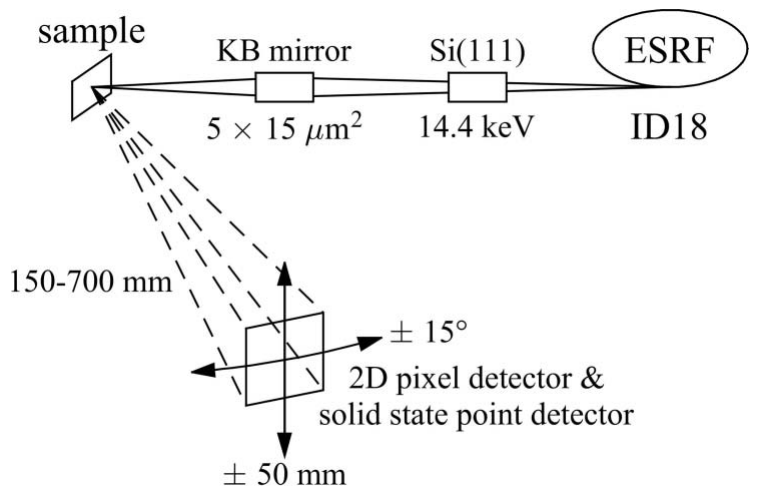

Figure 2

Experimental setup. A focused monochromatic synchrotron X-ray beam was used to excite atoms in the sample. The emitted fluorescent radiation and its modulation due to interference were recorded with a pixel detector. The energy spectrum was monitored using a point detector. To increase the solid angle of the pattern detection, the detector assembly can be translated and rotated. 
via both lower and upper thresholds. Filters cannot be used for this purpose, as appropriate suppression of the primary radiation could be achieved only for low energy differences with impractically thick filters causing huge intensity loss. Also the possible inhomogeneity of the filters can appear on the detected images.

It is clear from the above that from the point of view of structure determination one should measure as large a solid angle as possible to collect many Kossel lines, while from the point of view of determining the fine structure of lines one needs high angular resolution. To obtain good contrast in the Kossel pattern one needs either as perfect crystals as possible or detectors that have a large dynamic range to handle the low contrast obtainable from non-perfect crystals.

The first two requirements lead to a huge number of pixels. Let us estimate the parameters of the detector. Today's hybrid pixel detectors have typically $50 \mu \mathrm{m} \times 50 \mu \mathrm{m}$ pixels. Taking $0.001^{\circ}$ angular resolution, the minimum distance between sample and detector is about $2 \mathrm{~m}$. So, the number of pixels on a detector covering a half sphere is $\sim 10^{10}$. That is absolutely unrealistic at the present level of technology. Two-dimensional detectors with $\sim 10^{6}$ pixels are readily available. This means that we have to relax the requirements. Either we measure in a smaller solid angle, or degrade resolution, or some compromise in between.

\section{Experimental setup}

In our setup (Fig. 2), we used a $512 \times 512$ hybrid pixel detector capable of direct X-ray detection. It is based on a Medipix-2 chip developed at CERN and bonded to a $300 \mu \mathrm{m}$-thick Si sensor (Hatsui \& Graafsma, 2015). Placing the detector at about $500 \mathrm{~mm}$ from the sample covers only a $3^{\circ} \times 3^{\circ}$ solid angle. We extended this up to $12^{\circ} \times 12^{\circ}$ by rotating the detector about a vertical axis centered on the sample and translating it parallel to this axis (see Fig. 2). This setup was built at the ID18 beamline of the ESRF (Rüffer \& Chumakov, 1996). The primary $14.4 \mathrm{keV}$ X-ray beam was monochromated to $\sim 2 \mathrm{eV}$ bandwidth using a $\mathrm{Si}$ (111) channel-cut monochromator. A Kirkpatrick-Baez mirror (Kirkpatrick \& Baez, 1948) focused the beam to a $15 \mu \mathrm{m} \times 5 \mu \mathrm{m}$ spot on the surface of the sample. The incident intensity on the sample was $\sim 10^{14}$ photons $\mathrm{s}^{-1}$. Taking into account the fluorescent yield, geometrical factors and absorption we expect $\sim 10^{4}$ photons per pixel per second to the detector. Statistically this would be enough to see the Kossel lines even if the contrast is degraded. However, in practice one cannot see them and we found that the reason is the non-uniform response of the detector. Even after using the flat-field correction of the detector (via individual pixel threshold offsets) the statistical fluctuation is about two orders of magnitude larger than that corresponding to the Poisson noise (Fig. 3). To make the patterns visible, we need custom data processing described in the next section.

Beside fluorescent radiation, X-ray photons from other processes, such as Compton scattering from the air and from the sample, and elastic scattering from the air and from the surrounding objects (such as detector arms shielding, etc.), can

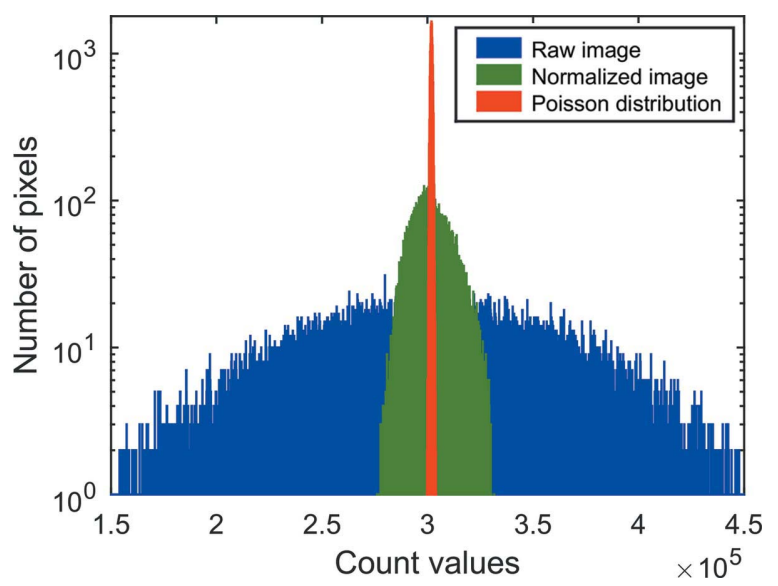

Figure 3

Statistical properties of detector response to a homogeneous illumination. Distribution of an as-measured pattern (wide blue part), normalized pattern (narrower green part) and the ideal Poisson distribution (narrow red central part).

also reach the detector. These give an additional background, which might further degrade the contrast of the Kossel lines. Part of these contributions can be avoided by proper shielding. Those parts, which cannot be suppressed, should be accounted for in the evaluation process. In order to measure these background contributions, we installed a small solid-state detector close to the two-dimensional detector. Using this, we monitored the energy distribution of the radiation reaching the two-dimensional detector and could identify the amount of radiation originating from the various processes.

\section{Pattern processing}

To correct for the inhomogeneity of the detector we first applied a normalization procedure as follows: we accumulated a pattern of homogeneous or spatially slowly varying intensity distribution (normalizer pattern, $N$ ) under similar conditions as the pattern of the sample under study (pattern to normalize, $P$ ). It can be done either by spinning the sample about an axis that does not intersect the detector (to avoid a fixed point) or by using an amorphous material containing the same kind of fluorescent atoms as the sample. After dividing pixel-by-pixel the two patterns $(K=P / N)$, the inhomogeneous sensitivity pattern characteristic to the detector disappears and sharp features, such as the Kossel lines, become visible. During this normalization the slowly varying component of the fluorescent background is removed that we are not interested in anyway. To further improve the visibility of the Kossel lines we made this background intensity constant by fitting a low-order polynomial function to the normalized patterns $(S=$ lowfrequency fit of $K$ ) and then calculated $K / S$, the final pattern. This brings the base fluorescent intensity to one and transforms the Kossel patterns to their natural intensity scale. We also made minor corrections to the patterns by replacing dead and hot pixel values by the average of their surrounding pixels. This whole procedure gives a significant improvement in the 
statistical properties of the patterns, but the pixel-to-pixel fluctuation remains larger than the Poisson noise by one order of magnitude (Fig. 3).

The next step in the evaluation is a description of the lines. For a given direct (and reciprocal) lattice, an orientation matrix and fluorescent wavelength, the direction of the Kossel cones for each set of planes can be calculated (Fig. 1). With appropriate instrumental parameters (sample-to-detector distance, detector size, detector scan parameters, detector tilt angles, sample displacements etc.) one can also locate the measured images in the same space. We developed a software that draws the Kossel cones on top of the preprocessed patterns and allows interactive adjustment or refinement of all structural and instrumental parameters. Once the geometry of the experiment is perfectly described, this software is also capable of extracting the Kossel line profiles for each (well and less visible) reflection by averaging measured intensities along narrow concentric cones of the given Kossel cone.

\section{Case of $\mathrm{NiO}$}

In the following we show a typical measurement on a singlecrystal of $\mathrm{NiO}$. The raw measured images of a $4 \times 3$ position scan of the detector containing $2 \times 2$ chips of $256 \times 256$ pixels is shown in Fig. 4(a). Kossel lines can barely be seen; the image is dominated by different sensitivity of the chips. To process these images we used our software. The normalized, background eliminated and aligned pattern $(K / S)$ is depicted in Fig. 4(b). Now the Kossel pattern is clearly visible. The intensity scale and the characteristic profile widths indicate approximately two orders of magnitude weaker and wider Kossel lines than theoretically expected. We interpret this mostly by the non-perfection of the crystal. Still, the lightdark fine structure caused by the interference between the direct and the Bragg reflected beam is clearly visible. The pattern was indexed and oriented with the face-centered cubic lattice of $\mathrm{NiO}$ as shown in Fig. 4(c). Note that for clarity only cones for $K \bar{\alpha}$ wavelength are plotted. Line profiles were extracted for all lines. Here, as an illustration, the profiles of the (111), (002), (022), (113), (040) and (402) reflections are shown in Fig. 4(d). NiO is a centrosymmetric structure and the origin of the conventional cell is located on the inversion center. Therefore, all reflections have either $0^{\circ}$ or $180^{\circ}$ phases. $\mathrm{Ni}$ atoms are also located on the origin, which determines the zero phase for the Kossel lines. According to the theory (Hutton et al., 1985), the minimum-then-maximum profile (with increasing angle) corresponds to a phase of $0^{\circ}$, while the maximum-then-minimum profile corresponds to a phase of $180^{\circ}$. This characteristic feature allows us to determine the phase by qualitative inspection of the line profile. Seeing that all of our profiles take a minimum before a maximum, we can conclude that all phases are zero in this structure. This agrees perfectly with the phases of calculated structure factors. Beside the overall light-dark structure of the lines, the precise profile depends on many experimental factors, such as the sample geometry, the crystal quality, the spatial resolution (a)

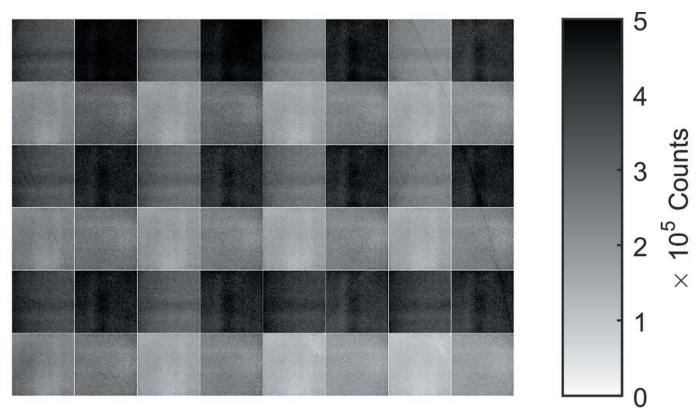

(b)

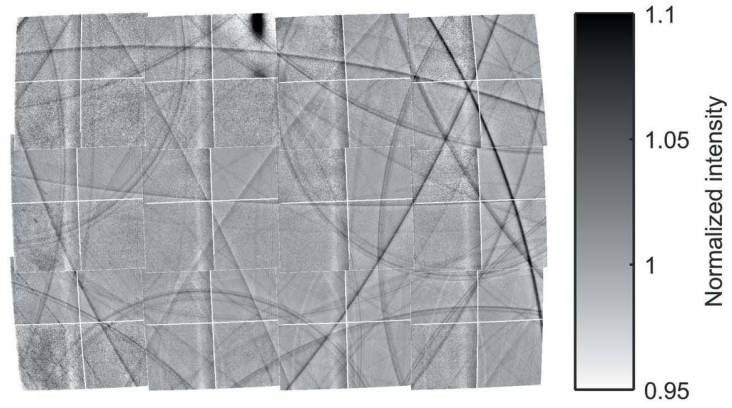

(c)

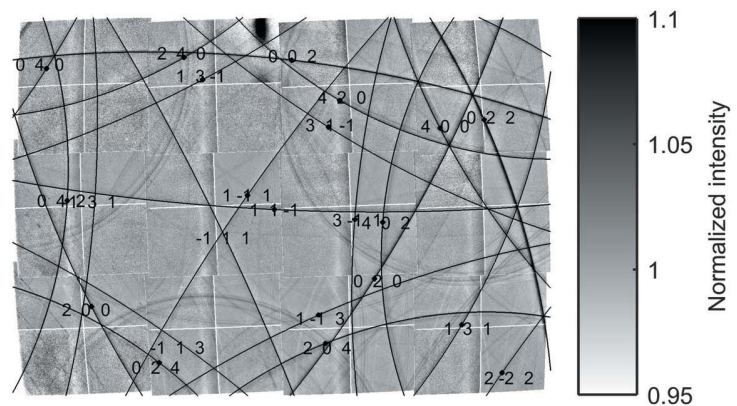

$(d)$

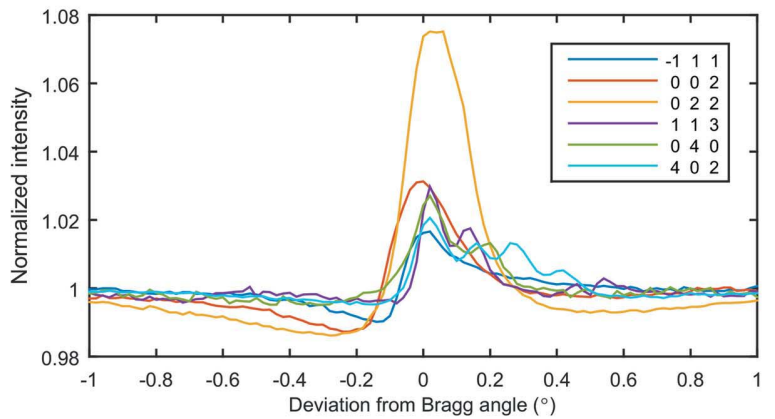

(e)

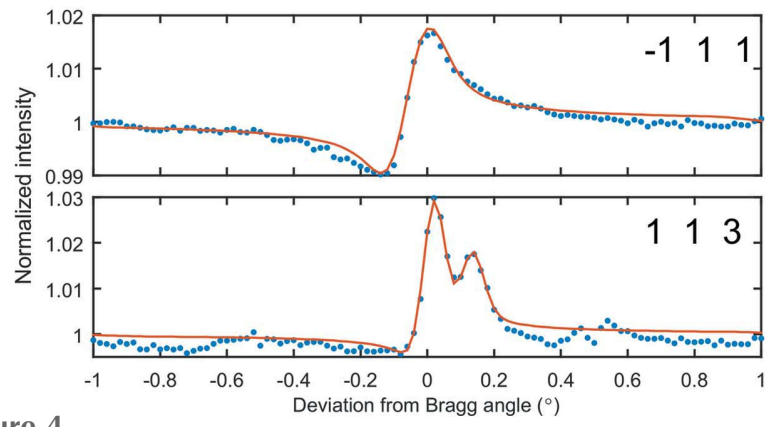

Figure 4

Kossel pattern of a NiO single-crystal. (a) Raw, unprocessed image shows barely visible lines. (b) The normalized, background eliminated and aligned images reveal an extended pattern about the threefold symmetry axis of the cubic structure. (c) Indexing the lines allows refinement of various structural and experimental parameters. $(d)$ Kossel line profiles are extracted for several reflections as a function of the deviation from the Bragg angle. $(e)$ Refinement of calculated Kossel line profiles yields $0 \pm 10^{\circ}$ phases for the structure factors. 


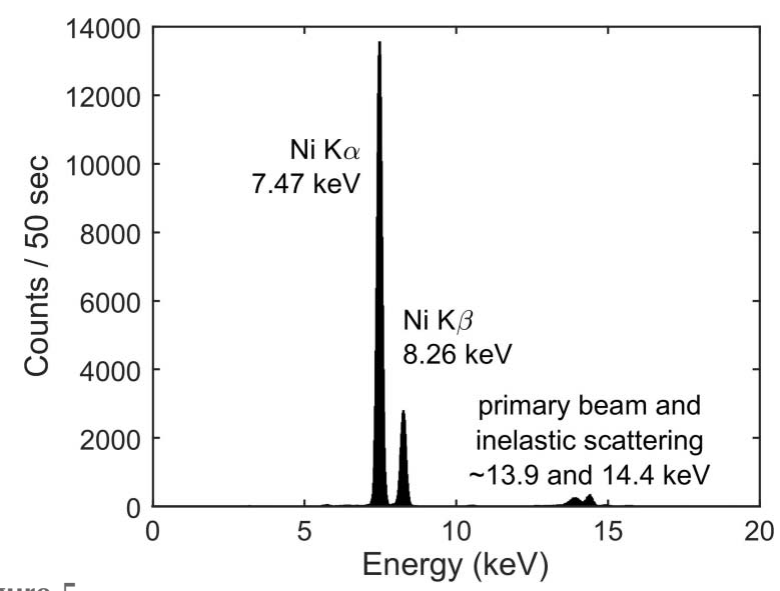

Figure 5

Energy spectrum of the X-rays reaching the detectors. The most prominent part is the Ni fluorescent radiation; however, there is a small higher-energy contribution from the incident beam and its inelastic scattering.

and efficiency of the detector, and the energy of the exciting radiation. The quantitative fitting of the line shape is very involved and it is out of the scope of the present article. However, we show here two fitted line profiles for illustration in Fig. 4(e), without detailed theoretical discussion. The calculations are based on the theory worked out by Hutton et al. (1985), Hannon \& Trammell (1969) and Hannon et al. $(1974 a, b)$. In both cases, the phase converged to the expected $0^{\circ}$ value within $10^{\circ}$. Compared with the large smearing of the theoretical lines this agreement is reasonable.

Finally we show the energy spectrum recorded simultaneously with a Kossel pattern (Fig. 5). Besides the Ni $K_{\alpha}$ and $K_{\beta}$ emission lines ( $7.47 \mathrm{keV}$ and $8.26 \mathrm{keV}$, respectively), we see two more contributions: one at $14.4 \mathrm{keV}$ and a wider highintensity region at about $13.9 \mathrm{keV}$. These high-energy contributions correspond to the exciting incident beam and the inelastic scattering from the air and from the sample itself. The identification of the above non-fluorescent contributions facilitates the description of background in the fitting procedure.

\section{Summary}

A setup for the measurement of Kossel line patterns has been described. This allows the collection of synchrotron-radiationexcited patterns to be made. It was shown that it is essential to measure normalizer patterns to compensate for the nonuniform response of the detector. It was illustrated that even if the contrast of the Kossel lines is degraded, and the width is increased compared with the theoretically expected values due to non-perfection of the crystals and other experimental factors, the light-dark fine structure of the lines can be readily obtained from the measured pattern. This type of apparatus opens the way for very fast collection of a single-crystal data set, including phase information and for taking single-crystal data at extremely non-ambient conditions. The quantitative determination of the phase of various reflections using a fitting procedure for more complicated structures in which the fluorescent atoms are not at inversion centers is beyond the scope of this paper. For these cases, theoretical and technical considerations will be given in a separate publication.

\section{Acknowledgements}

The authors thank the ESRF for the beam time and for the efficient help of the ID18 beamline staff. This work has been supported by the OTKA 105691 grant.

\section{References}

Cowley, J. M. (1964). Acta Cryst. 17, 33-40.

Hannon, J. P., Carron, N. J. \& Trammell, G. T. (1974a). Phys. Rev. B, 9, 2791-2809.

Hannon, J. P., Carron, N. J. \& Trammell, G. T. (1974b). Phys. Rev. B, 9, 2810-2831.

Hannon, J. P. \& Trammell, G. T. (1969). Phys. Rev. 186, 306-325.

Hatsui, T. \& Graafsma, H. (2015). IUCrJ, 2, 371-383.

Hutton, J. T., Trammell, G. T. \& Hannon, J. P. (1985). Phys. Rev. B, 31, 743-751.

Kirkpatrick, P. \& Baez, A. V. (1948). J. Opt. Soc. Am. 38, 766.

Kossel, W., Loeck, V. \& Voges, H. (1935). Z. Phys. 94, 139-144.

Lider, V. V. (2011). Crystallogr. Rep. 56, 169-189.

Rüffer, R. \& Chumakov, A. I. (1996). Hyperfine Interact. 97-98, 589604. 